\title{
INDECOMPOSABLE HILBERT-SCHMIDT OPERATORS
}

\author{
GARY WEISS
}

\begin{abstract}
In 1973, L. G. Brown, R. G. Douglas, and P. A. Fillmore characterized the set of all operators of the form $N+K$ where $N$ is a normal operator and $K$ is a compact operator and they asked whether or not every Hilbert-Schmidt operator is the sum of a normal operator and a trace class operator. They later asked if, for every Hilbert-Schmidt operator $A$, there exists a normal operator $N$ for which $A \oplus N$ is the sum of a normal operator and a trace class operator. We produce a large class of HilbertSchmidt operators $A$ none of which is the sum of a normal operator and a trace class operator, and furthermore, for each arbitrary operator $Q, A \oplus Q$ is not the sum of a normal operator and a trace class operator. We then use this to show that their characterization of the operators $N+K$ does not hold true if we replace the class of compact operators by the trace class or by any ideal $I$ for which $I \neq I^{1 / 2}$. In the case of the trace class, we show that even if the vanishing of the Helton and Howe trace invariant were added to the hypothesis of their characterization, it would not hold true.
\end{abstract}

Let $H$ be a separable, infinite-dimensional complex Hilbert space. Let $L(H)$ denote the algebra of all bounded linear operators on $H$, and let $K(H)$ denote the two-sided ideal in $L(H)$ of all compact operators. Furthermore, let $C_{2}$ and $C_{1}$ be the Hilbert-Schmidt and trace class ideals, respectively, of compact operators in $L(H)$, and let $(N)$ be the class of normal operators in $L(H)$. Finally, for each bounded operator $A$, let $\sigma_{e}(A)$ denote the essential spectrum of $A$.

L. G. Brown, R. G. Douglas, and P. A. Fillmore characterized $(N)+$ $K(H)$ by proving [2, Theorem 11.2] that an operator $A$ is decomposable into the sum of a normal operator and a compact operator (i.e. $A \in(N)+K(H)$ ) if and only if $A^{*} A-A A^{*} \in K(H)$ and index $(A-\lambda I)=0$ for every $\lambda$ $\notin \sigma_{e}(A)$.

One then asks under what circumstances the ideal of compact operators can be replaced by the ideal $C_{1}$ in this result. Indeed, if $A \in(N)+C_{1}$, then $A$ not only satisfies the two conditions:

(1) $A^{*} A-A A^{*} \in C_{1}$ and

(2) index $(A-\lambda I)=0$ for every $\lambda \notin \sigma_{e}(A)$, but in addition, the trace of $A^{*} A-A A^{*}$ is clearly 0 . In fact, more must be true. If $A \in(N)+C_{1}$, then the Helton and Howe trace invariant [4] vanishes for $A$. This follows since if

Presented to the Society, January 25, 1975; received by the editors October 17, 1974 and, in revised form, July $28,1975$.

AMS (MOS) subject classifications (1970). Primary 47A55, 47B05, 47B10; Secondary 47B47, 15A60, 47A65.

Key words and phrases. Hilbert-Schmidt operator, trace class operator, $N+K$, decomposable, ideal, $2 \times 2$ operator-valued matrix, weighted shift operator. 
$A=N+C$ where $N$ is normal and $C \in C_{1}$, then the trace invariant for $N+C$ is the same as that for $N$, and the trace invariant of a normal operator vanishes. One then asks

Question 1. Is it true that ' $A \in(N)+C_{1}$ if and only if (1) $A^{*} A-A A^{*} \in$ $C_{1}$, (2) index $(A-\lambda I)=0$ for every $\lambda \notin \sigma_{e}(A)$, and (3) $A$ has vanishing Helton and Howe trace invariant'?

Brown, Douglas and Fillmore conjectured that this is not the case, and in this connection they posed the following question in [2, pp. 123-124] and at the 1973 Wabash International Conference on Banach Spaces.

Question 2. Prove that not every Hilbert-Schmidt operator is decomposable into the sum of a normal operator and a trace class operator (i.e., $\left.C_{2} \not \subset(N)+C_{1}\right)$.

An affirmative solution to Question 2 answers Question 1 in the negative. This follows from the facts that any Hilbert-Schmidt operator $A$ for which $A \notin(N)+C_{1}$ nevertheless satisfies conditions (1)-(3) in Question 1. That conditions (1) and (2) hold for every Hilbert-Schmidt operator is well known. That condition (3) holds for every Hilbert-Schmidt operator follows from the fact that every compact operator with a trace class self-commutator has a vanishing Helton and Howe trace invariant.

They also asked the following related question.

Question 3. If $A$ is a Hilbert-Schmidt operator, does there exist a normal operator $N$ such that $A \oplus N \in(N)+C_{1}$ ?

In what follows, we answer all three questions and generalize the results. In particular, we answer Question 1 in the negative by proving that $C_{2} \not \subset(N)+$ $C_{1}$, thereby solving Question 2. For this we produce a large class of operators $A$ in $C_{2}$ that are not contained in $(N)+C_{1}$. Furthermore we show that each such $A$ in $C_{2}$ leads to a solution, in the negative, of Question 3. In fact, we show that for each such $A$ in $C_{2}$ and every $Q \in L(H)$ ( $Q$ need not be normal) we obtain $A \oplus Q \notin(N)+C_{1}$. Our techniques apply to more general ideals and not merely to $C_{2}$ and $C_{1}$. We shall state and prove our results in this more general setting.

Preliminary remarks. We shall use $I$ and $J$ to denote ideals. One should keep in mind that in relation to the above, $J$ replaces $C_{2}$ and $I$ replaces $C_{1}$.

Let $I^{2}$ denote the ideal generated by $I \cdot I$. Note the well-known and easily verified facts that $|T|^{2} \in I^{2}$ if and only if $|T| \in I$, and $|T| \in I$ if and only if $T \in I$. Let $I^{1 / 2}$ denote the unique ideal whose square is $I$.

If $H_{1}$ and $H_{2}$ are two Hilbert spaces of the same dimension, then there are many isometric isomorphisms $U$ (i.e., unitary transformations) mapping $H_{1}$ onto $H_{2}$. Each such unitary transformation $U$ induces a canonical ${ }^{*}$-isometric isomorphism $F_{U}$ mapping $L\left(H_{1}\right)$ onto $L\left(H_{2}\right)$ via the map $F_{U}: A \rightarrow U A U^{-1}$. It is clear that each such induced $F_{U}$ preserves compactness, positivity, and eigenvalues (counting multiplicities). From this it is clear that each such induced $F_{U}$ takes each ideal in $L\left(H_{1}\right)$ into the unique ideal in $L\left(H_{2}\right)$ that has the same Calkin ideal set [3]. That is, we induce a multiplicative lattice isomorphism between the lattice of ideals in $L\left(H_{1}\right)$ and the lattice of ideals in $L\left(\mathrm{H}_{2}\right)$. What is more, this lattice isomorphism is the same for all such $F_{U}$. Hence we can identify ideals in $L\left(H_{1}\right)$ with ideals in $L\left(H_{2}\right)$. In this paper, any ideal contained in $L(H)$, or $L(H \oplus H)$, or $L(H \oplus H \oplus H)$ will be thought 
of as simultaneously lying in $L(H), L(H \oplus H)$, and $L(H \oplus H \oplus H)$.

Let $M\left(T_{11}, T_{12}, T_{21}, T_{22}\right)$ denote the operator in $L(H \oplus H)$ which is represented by the $2 \times 2$ matrix with operator entries $T_{i j}$ in the $(i, j)$ position, for $i, j=1,2$. It is easy to show that if $I$ is an ideal in $L(H)$, and so, under our identification, $I$ is an ideal in $L(H \oplus H)$, then $I$, considered to be in $L(H \oplus H)$, is precisely $\left\{M\left(T_{11}, T_{12}, T_{21}, T_{22}\right): T_{i j} \in I\right.$ for $\left.i, j=1,2\right\}$.

We now state and prove our results.

Theorem 1 (The main theorem). If $I$ is an ideal in $L(H)$ and $A \notin I$, then

$$
M(0,0, A, 0)=\left(\begin{array}{cc}
0 & 0 \\
A & 0
\end{array}\right) \notin(N)+I .
$$

Proof. Suppose to the contrary that $A \notin I$ and yet $M(0,0, A, 0) \in(N)+$ I. Then $M(0,0, A, 0)=N-M(X, Y, S, T)$ for some normal operator $N \in L(H \oplus H)$ and operators $X, Y, S, T \in I$ where $I$ is considered to be in $L(H)$. Then $N=M(X, Y, A+S, T)$ and $N^{*}=M\left(X^{*},(A+S)^{*}, Y^{*}, T^{*}\right)$. Since $N$ is normal, $N^{*} N=N N^{*}$. Substituting and computing using the last two equations, we obtain the equation

$$
M\left(X^{*} X+(A+S)^{*}(A+S), \cdot, \cdot, \cdot\right)=M\left(X X^{*}+Y Y^{*}, \cdot, \cdot, \cdot \cdot\right) .
$$

Therefore, $X^{*} X+(A+S)^{*}(A+S)=X X^{*}+Y Y^{*}$ or, equivalently, $|A+S|^{2}=\left|X^{*}\right|^{2}+\left|Y^{*}\right|^{2}-|X|^{2}$. Since $X, Y \in I$, and since every ideal is closed under the operation of taking adjoints, we obtain $\left|X^{*}\right|^{2},\left|Y^{*}\right|^{2},|X|^{2} \in I^{2}$, and therefore $|A+S|^{2} \in I^{2}$. Hence $|A+S| \in I, A+S \in I$, and finally $A \in I$, which contradicts our assumption that $A \notin I$. Q.E.D.

Corollary. If $I$ and $J$ are ideals in $L(H)$ for which $J \not \subset I$, then $J \not \subset(N)+$ I. In particular, $C_{2} \not \subset(N)+C_{1}$.

Proof. For every $A \in J \backslash I, M(0,0, A, 0) \in J$ and by Theorem 1 , $M(0,0, A, 0) \notin(N)+I$. Therefore $J \not \subset(N)+I$. Q.E.D.

THeOREM 2. If $I$ is an ideal in $L(H)$ and $A \notin I$, then $M(0,0, A, 0) \oplus Q$ $\notin(N)+I$ for every $Q \in L(H)$.

Proof. Use the proof of Theorem 1, using $3 \times 3$ matrices with operator entries to represent operators in $L(H \oplus H \oplus H)$ in place of the $2 \times 2$ matrices. As in the proof of Theorem 1, apply the equation $N^{*} N=N N^{*}$ and after computing, consider only the $(1,1)$ position. Q.E.D.

Corollary 1. If $A \in C_{2} \backslash C_{1}$, then $M(0,0, A, 0) \in C_{2}$, but for every $Q \in L(H), M(0,0, A, 0) \oplus Q \notin(N)+C_{1}$.

Proof. This follows trivially from Theorem 2. Q.E.D.

Let us now reformulate Question 1 for an arbitrary ideal $I$ and ask another related question.

Question 4a. Is it true that ' $A \in(N)+I$ if and only if $A^{*} A-A A^{*} \in I$ and index $(A-\lambda I)=0$ for every $\lambda \notin \sigma_{e}(A)$ '?

Question 4b. In the case $I \subset C_{1}$, is it true that ' $A \in(N)+I$ if and only if $A^{*} A-A A^{*} \in I$, index $(A-\lambda I)=0$ for every $\lambda \notin \sigma_{e}(A)$, and the Helton and Howe trace invariant vanishes for $A^{\prime}$ ?

We stated earlier that $I=K(H)$ solves Question 4a in the affirmative. It is 
the only ideal we know that solves Question 4a in the affirmative. However, the next corollary yields many ideals which solve Question $4 \mathrm{a}$ in the negative.

Corollary 2. If $I$ is an ideal for which $I \neq I^{1 / 2}$, then it is false that ' $A \in(N)+I$ if and only if $A^{*} A-A A^{*} \in I$ and $\operatorname{index}(A-\lambda I)=0$ for every $\lambda \notin \sigma_{e}(A)$ '.

Proof. Choose $A \in I^{1 / 2} \backslash I$ and let $A_{1}=M(0,0, A, 0)$. Then $A_{1} \in I^{1 / 2}$ and so $A_{1}^{*} A_{1}-A_{1} A_{1}^{*} \in I$ and index $\left(A_{1}-\lambda I\right)=0$ for every $\lambda \notin \sigma_{e}\left(A_{1}\right)$. But by Theorem $1, A_{1} \notin(N)+I$. Q.E.D.

A point of view. The point of view which led to these solutions motivates the following question, which may be important in decomposition theory. Is every Hilbert-Schmidt weighted shift operator of finite multiplicity decomposable into the sum of a normal operator and a trace class operator? Note that our Hilbert-Schmidt operator $M(0,0, A, 0)$ seems far from a weighted shift operator of finite multiplicity in that its nonzero entries lie 'far' from the diagonal. There appears to be an important theme arising here. Loosely speaking, some operators whose nonzero entries are near or on the diagonal are not unitarily equivalent to operators whose nonzero entries are far from the diagonal, and some are. Which ones are and which ones are not appears, at times, to be the central issue. This theme has arisen before in regard to commutators (operators of the form $A B-B A$ ). Often, diagonal operators are not commutators or they present difficult commutator problems (see [5]), whereas operators of the form $M(0,0, A, 0)$ are easily written as the right kind of commutators. It is becoming well known that entries on the diagonal are harder to handle than entries off the diagonal, in some contexts. J. H. Anderson makes some of the same observations in [1, Remark 4.4].

The next theorem answers the previous question in the negative, but leads to two other questions. We give two proofs of this theorem. The first one is a technique which can be used to obtain a more general result. The second one cannot, but it is shorter and depends on Theorem 1 .

THEOREM 3. If $U$ is the unilateral weighted shift operator with weights $\left(w_{n}\right)$ where $w_{2 n-1}=n^{-3 / 4}$ and $w_{2 n}=0$ for every $n$, then $U \in C_{2}$ and $U \notin(N)+$ $C_{1}$.

Proof I. Clearly $U \in C_{2}$. Suppose to the contrary that $U=N+C$ where $N$ is a normal operator and $C \in C_{1}$. Then $N=U-C$ and

$$
\begin{aligned}
0=N^{*} N-N N^{*}= & \left(U^{*} U-U U^{*}\right)-\left(U^{*} C-C U^{*}\right) \\
& -\left(C^{*} U-U C^{*}\right)+\left(C^{*} C-C C^{*}\right),
\end{aligned}
$$

and hence

$$
U^{*} U-U U^{*}=\left(U^{*} C-C U^{*}\right)+\left(C^{*} U-U C^{*}\right)-\left(C^{*} C-C C^{*}\right) .
$$

It is well known that the product of an operator in $C_{2}$ and an operator in $C_{1}$ is an operator in $C_{2 / 3}$. Therefore the right-hand side of the previous equation is in $C_{2 / 3}$, and so $U^{*} U-U U^{*} \in C_{2 / 3}$. However, by computing we see that $U^{*} U-U U^{*}$ is the diagonal operator with entries $\left|w_{1}\right|^{2},\left|w_{2}\right|^{2}-\left|w_{1}\right|^{2}$, $\left|w_{3}\right|^{2}-\left|w_{2}\right|^{2}, \ldots$ Hence, if we choose $w_{2 n-1}=n^{-3 / 4}$ and $w_{2 n}=0$ for every $n$, then by computing we see that $U^{*} U-U U^{*}$ is the diagonal operator with 
diagonal entries $\left(1,-1,2^{-3 / 2},-2^{-3 / 2}, 3^{-3 / 2},-3^{-3 / 2}, \ldots\right)$, which is not contained in $l^{2 / 3}$. Therefore $U^{*} U-U U^{*} \notin C_{2 / 3}$, which is a contradiction.

Proof II. It is easy to show that since $w_{2 n}=0$ for every $n, U$ is unitarily equivalent to $M(0,0, D, 0)$ where $D$ is the diagonal matrix whose entries are the numbers $w_{2 n-1}$. Hence $D \notin C_{1}$. Therefore, by Theorem $1, U \notin(N)+$ $C_{1}$. Q.E.D.

The following two questions are concerned with characterizing $(N)+C_{1}$.

Question 5. Which Hilbert-Schmidt weighted shift operators are contained in $(N)+C_{1}$ ?

Question 6. Does there exist a Hilbert-Schmidt weighted shift operator which is not contained in $C_{1}$ but which is contained in $(N)+C_{1}$ ?

The answer to Question 6 is yes. I. D. Berg solved this, and we give a simple version of his proof.

Let $U_{n}$ be the unilateral shift on the $n$-dimensional Hilbert space $H$ $=\operatorname{span}\left\{e_{k}\right\}_{k=1}^{n}$. Let $K_{n}$ be the $n \times n$ matrix for which $K_{n} e_{k}=0$ if $1 \leqslant k$ $\leqslant n-1$, and $K_{n} e_{n}=-e_{1}$. It is clear then that $V_{n}=U_{n}-K_{n}$ is an $n \times n$ matrix in which every row and every column has precisely one nonzero entry, and that entry is 1 . It follows that $V_{n}$ is unitary. Also the $C_{p}$-norm $(1 \leqslant p$ $<\infty)$ of $K_{n}=U_{n}-V_{n}$ is 1 and that of $U_{n}=V_{n}+K_{n}$ is $(n-1)^{1 / p}$. Letting $N=\Sigma \oplus n^{-2} V_{n}, S=\Sigma \oplus n^{-2} U_{n}$, and $K=\Sigma \oplus n^{-2} K_{n}$, we see that $N$ is normal and $S \in C_{2} \backslash C_{1}$ and is a weighted shift. Also $S=N+K$ and $\|K\|_{C_{1}}=\sum n^{-2}<\infty$.

Question 5 remains unsolved. To this end Theorem 3 and the affirmative solution to Question 6 may be a beginning.

\section{REFERENCES}

1. J. H. Anderson, Derivations, commutators, and the essential numerical range, Dissertation, Indiana University, 1971.

2. L. G. Brown, R. G. Douglas and P. A. Fillmore, Unitary equivalence modulo the compact operators and extensions of $C^{*}$-algebras, Lecture Notes in Math., vol. 345, Springer-Verlag, Berlin and New York, 1973, pp. 118, 123-124.

3. J. W. Calkin, Two-sided ideals and congruences in the ring of bounded operators in Hilbert space, Ann. of Math. (2) 42 (1941), 839-873. MR 3, 208.

4. J. William Helton and Roger E. Howe, Integral operators: traces, index, and homology, Lecture Notes in Math., vol. 345, Springer-Verlag, Berlin and New York, 1973.

5. Gary Weiss, Commutators and operator ideals, Dissertation, University of Michigan, 1975.

Department of Mathematics, University of Michigan, Ann Arbor, Michigan 48104

Current address: Department of Mathematics, University of Cincinnati, Cincinnati, Ohio 45221 